\title{
Cotunneling theory of atomic spin inelastic electron tunneling spectroscopy
}

\author{
F. Delgado and J. Fernández-Rossier \\ Departamento de Física Aplicada, Universidad de Alicante, E-03690 San Vicente del Raspeig, Spain
}

(Received 21 March 2011; revised manuscript received 24 May 2011; published 19 July 2011)

\begin{abstract}
We propose cotunneling as the microscopic mechanism that makes possible inelastic electron tunneling spectroscopy of magnetic atoms in surfaces for a wide range of systems, including single magnetic adatoms, molecules, and molecular stacks. We describe electronic transport between the scanning tip and the conducting surface through the magnetic system (MS) with a generalized Anderson model, without making use of effective spin models. Transport and spin dynamics are described with an effective cotunneling Hamiltonian in which the correlations in the magnetic system are calculated exactly and the coupling to the electrodes is included up to second order in the tip MS and MS substrate. In the adequate limit our approach is equivalent to the phenomenological Kondo exchange model that successfully describes the experiments. We apply our method to study in detail inelastic transport in two systems, stacks of cobalt phthalocyanines and a single $\mathrm{Mn}$ atom on $\mathrm{Cu}_{2} \mathrm{~N}$. Our method accounts for both the large contribution of the inelastic spin exchange events to the conductance and the observed conductance asymmetry.
\end{abstract}

DOI: 10.1103/PhysRevB.84.045439

PACS number(s): 75.30.Et, 74.55.+v, 71.10.Fd

\section{INTRODUCTION}

The combination of two powerful techniques, inelastic electron tunneling spectroscopy (IETS) and scanning tunneling microscopy (STM) makes it possible to probe inelastic excitations with subatomic resolution. The STM-IETS technique was first applied to the study of vibrational excitations of single molecules on surfaces ${ }^{1}$ and has more recently been used to study spin excitations of a single and a few magnetic atoms and molecules deposited on surfaces. ${ }^{2-13}$ In STM-IETS, electrons tunnel between the tip and the conducting substrate going through the magnetic system. As the bias voltage $V$ is increased, a new conduction channel opens whenever $|e V|$ is larger than the energy of some internal excitation of the atom, which results in a stepwise increase of the differential conductance $d I / d V$ and a peak or dip in the $d^{2} I / d V^{2}$. Tracing the evolution of the elementary excitations as a function of an applied magnetic field and fitting to effective spin Hamiltonians permits one to infer the single-ion magnetic anisotropy tensor as well as exchange coupling between adjacent atoms and molecules. ${ }^{2-12}$

The IETS-STM technique has been applied to a variety of magnetic systems weakly coupled to a conducting substrate. The list includes a single transition metal atom ( $\mathrm{Mn}, \mathrm{Fe}, \mathrm{Co}$ ) deposited on a single monolayer of $\mathrm{Cu}_{2} \mathrm{~N}$ on copper, ${ }^{4-7,13}$ to chains of up to $10 \mathrm{Mn}$ atoms on the same substrate, ${ }^{3}$ to $\mathrm{Fe}$-phthalocyanine $(\mathrm{FePc})$ molecules on oxidized $\mathrm{Cu},{ }^{9}$ to stacks of CoPc molecules on $\mathrm{Pb},{ }^{8,10}$ to $\mathrm{MnPc}$ on $\mathrm{PbO},{ }^{11}$ and, more recently, a single $\mathrm{Fe}$ atom on InSb, a semiconducting substrate. ${ }^{12}$ For all these systems it is possible to describe the spin exchange assisted tunneling, which accounts for the coupling between transport electrons and the localized spins of the magnetic atoms or molecules, with Kondo-like Hamiltonians. ${ }^{14-22}$ Whereas this approach successfully describes the main experimental results, including the differential conductance, as well as effects related to current-driven spin dynamics and/or a spin-polarized tip, there are questions that cannot be addressed using effective spin models:
(1) Why is the spin-assisted inelastic conductance comparable to the elastic contribution, in contrast with the phononassisted inelastic contribution?

(2) What is the microscopic origin of the spin exchange tunneling?

(3) Why is the inelastic conductance not always symmetric with respect to the inversion of the bias polarity?

In this work we provide a theoretical framework to model the existing STM-IETS experiments that addresses these questions. Our starting point is a generalized multiorbital and multisite Anderson model, in which the electrons in the localized orbitals of the magnetic system (MS) are hybridized to the itinerant states of the tip and the surface. The states of the MS are calculated by exact diagonalization of a microscopic Hamiltonian that can include Coulomb repulsion, crystal field, and spin-orbit coupling. Transport and spin dynamics are described by means of an effective cotunneling Hamiltonian in which the coupling to the tip and surface is included up to second order. This approach works provided that the charging energy of the MS is much larger than the temperature, applied bias potential, and electrode-induced broadening of the MS levels. Thus, the MS must be in the Coulomb blockade situation, where the charge is a good quantum number and current flows due to quantum charge fluctuations, known as cotunneling. ${ }^{23,24}$

When applied to a single-orbital Anderson model, the effective cotunneling Hamiltonian that we obtain is identical to the Kondo model obtained through the standard Schrieffer-Wolff transformation. ${ }^{25,26}$ Our method can be applied to systems with more than one localized orbital, necessary to address most experimentally relevant systems. ${ }^{2-13}$ The effective cotunneling Hamiltonian describes transitions between the different manybody states of the MS induced by their coupling to the itinerant electrons. This permits us to calculate the scattering rates, both for the dissipative dynamics of the spin excitations of the MS coupled to the leads and those leading to the current.

The rest of the paper is organized as follows. In Sec. II we present the derivation of the effective Hamiltonian and the procedure used to calculate the current, leaving some of the 
technical details for the appendices. In Sec. III we apply our approach to the case of a single-site Anderson model, which permits us to test our approach against well-established results. In Sec. IV we implement our approach to model transport through stacks of CoPc molecules. ${ }^{8,10}$ For that matter, we describe the CoPc stacks by means of a Hubbard model. In Sec. $\mathrm{V}$ we study the case of a single $\mathrm{Mn}$ adatom on a $\mathrm{Cu}_{2} \mathrm{~N}$ surface, ${ }^{3,6}$ using a multiorbital Anderson model where Coulomb interaction, crystal field, and spin-orbit coupling in the MS are included in the Hamiltonian and treated exactly, by means of numerical diagonalization. In Sec. VI we summarize our main results.

\section{THEORY}

\section{A. Effective Hamiltonian}

We describe a magnetic system weakly coupled to two electrodes, denoted as tip $(T)$ and surface $(S)$ without loss of generality, using the following Hamiltonian:

$$
\mathcal{H}=\mathcal{H}_{T}+\mathcal{H}_{S}+\mathcal{H}_{\mathrm{MS}}+\mathcal{V}_{\text {tun }}
$$

Here $\mathcal{H}_{T}+\mathcal{H}_{S}$ correspond to the Hamiltonian of the two electrodes, $\mathcal{H}_{\mathrm{MS}}$ the magnetic system, and $\mathcal{V}_{\text {tun }}$ the tunneling Hamiltonian. We shall consider the two electrodes as free electron reservoirs, i.e., $\mathcal{H}_{T}+\mathcal{H}_{S}=\sum_{\alpha} \epsilon_{\alpha} f_{\alpha}^{\dagger} f_{\alpha}$, where $f_{\alpha}^{\dagger}$ $\left(f_{\alpha}\right)$ is the creation (annihilation) operator of a quasiparticle with single-particle number $\alpha \equiv\{k, \eta, \sigma\}$, with momentum $k$, electrode $\eta=T, S$ and spin projection in the quantization direction $\sigma$. In general, the central region has a complicated many-body Hamiltonian that includes Coulomb repulsion, spin-orbit coupling, crystal field terms, and so on. The manybody eigenstates of $\mathcal{H}_{\mathrm{MS}},|q, n\rangle$, have a well-defined number of electrons $q$. Only 3 charge states, $q=q_{0}$, + , and - , are relevant. The $q_{0}$ corresponds to the ground state of the MS. The states + and - correspond to the MS with an extra electron $\left(q_{0}+1\right)$ and an extra hole $\left(q_{0}-1\right)$, respectively. The Hamiltonian of the isolated MS can be written as

$$
\mathcal{H}_{\mathrm{MS}}=\sum_{q, n} E_{q, n}|q, n\rangle\langle q, n| .
$$

The tunneling Hamiltonian is given by

$$
\mathcal{V}_{\text {tun }}=\sum_{\mathbf{i}, \alpha} V_{\alpha, \mathbf{i}} f_{\alpha}^{\dagger} d_{\mathbf{i}}+\text { H.c. }=\hat{\mathcal{V}}^{-}+\hat{\mathcal{V}}^{+},
$$

where the tunneling of electrons in and out of the MS are described by $\hat{\mathcal{V}}^{+}$and $\hat{\mathcal{V}}^{-}$, respectively. Here $d_{\mathbf{i}}^{\dagger}\left(d_{\mathbf{i}}\right)$ is the creation (annihilation) operator of an electron in a singleparticle state $\mathbf{i} \equiv\{i, \sigma\}$ with orbital quantum numbers $i$ and $\operatorname{spin} \sigma$. We assume that single-particle tunneling events are spin conserving and spin independent; i.e., $V_{k \eta \sigma, i \sigma^{\prime}}=V_{k \eta, i} \delta_{\sigma, \sigma^{\prime}}$.

We will start with the uncoupled Hamiltonian $\mathcal{H}_{0}=\mathcal{H}_{T}+$ $\mathcal{H}_{S}+\mathcal{H}_{\text {MS }}$. Since $\mathcal{H}_{0}$ commutes with the charge operator of the MS, the eigenstates of $\mathcal{H}_{0}$ can be labeled according to the charge $q$ in the central atom. We assume that the eigenvalues in the $q_{0}$ state are separated by a large gap from the states in the $q= \pm$ charge states, see Fig. 1 . In particular, the chemical potentials of the MS, defined as $\mu_{h}=E_{G}\left(N_{e}\right)-E_{G}\left(N_{e}-1\right)$ and $\mu_{e}=E_{G}\left(N_{e}+1\right)-E_{G}\left(N_{e}\right)$, with $E_{G}\left(N_{e}\right)$ the ground state energy corresponding to $N_{e}$ electrons, must satisfy $\left|\mu_{h}-\mu_{\eta}\right|,\left|\mu_{e}-\mu_{\eta}\right| \gg k_{b} T,|e V|$. This corresponds to the conditions of deep cotunneling in which the sequential firstorder transitions are exponentially suppressed. ${ }^{27-29}$ In this limit we can use degenerate perturbation theory to determine the dynamics of the states in the $q_{0}$ charge state, which we denote with $|N\rangle$. These states are tensor products of the electrode ground states and the many-body states $|q, n\rangle$ of the magnetic system. The tunneling operator (3) connects them to states $\left|M_{ \pm}\right\rangle$that are products of electrode states with 1 quasiparticle and MS states $\left|q_{0} \pm 1, m\right\rangle$. Unless otherwise stated, in the rest of the paper we label the MS island states with the shorthand notation $|n\rangle \equiv\left|q_{0}, n\right\rangle$ and $\left|m_{ \pm}\right\rangle=\left|q_{0} \pm 1, m\right\rangle$.

Using degenerate perturbation ${ }^{30}$ theory we can obtain an effective Hamiltonian for the $q_{0}$ charge state where the tunneling events are included to the lowest order:

$$
\mathcal{H}_{\text {cotun }}=\sum_{M_{\mp}} \frac{\mathcal{V}^{ \pm}\left|M_{\mp}\right\rangle\left\langle M_{\mp}\right| \mathcal{V}^{\mp}}{E_{M_{\mp}}-E_{0}}
$$

In the calculation of the effective Hamiltonian we are neglecting the energy variations of the unperturbed states inside the $q_{0}$ manifold, all taken to be $E_{0}$, compared to the charging energy. When expanding this operator in the basis of the electrode quasiparticles and the MS many-body states, we can write the effective Hamiltonian for the $q_{0}$ charge state as (see Appendix A for details)

$$
\mathcal{H}_{\text {cotun }}=\sum_{\alpha \alpha^{\prime}}\left[\hat{\mathcal{O}}_{\alpha \alpha^{\prime}}^{(+)}-\hat{\mathcal{O}}_{\alpha^{\prime} \alpha}^{(-)}\right] f_{\alpha}^{\dagger} f_{\alpha^{\prime}}+\sum_{\alpha} \hat{\mathcal{O}}_{\alpha \alpha}^{(-)},
$$

where

$$
\hat{\mathcal{O}}_{\alpha \alpha^{\prime}}^{ \pm} \equiv \sum_{n, n^{\prime}}\left\langle n\left|\hat{\mathcal{O}}_{\alpha \alpha^{\prime}}^{( \pm)}\right| n^{\prime}\right\rangle|n\rangle\left\langle n^{\prime}\right|
$$

are operators that act exclusively on the subspace $\left|q_{0}, n\right\rangle$ of the neutral MS. Their matrix elements read

$$
\left\langle n\left|\hat{\mathcal{O}}_{\alpha \alpha^{\prime}}^{(+)}\right| n^{\prime}\right\rangle=\sum_{i i^{\prime}, \sigma, \sigma^{\prime}, m_{+}} \frac{V_{\alpha, i} V_{\alpha^{\prime}, i^{\prime}}^{*}}{E_{m_{+}}-E_{0}-\epsilon_{\alpha}} \gamma_{n, n^{\prime}}^{m_{+}}\left(i i^{\prime}, \sigma \sigma^{\prime}\right)
$$

and

$$
\left\langle n\left|\hat{\mathcal{O}}_{\alpha \alpha^{\prime}}^{(-)}\right| n^{\prime}\right\rangle=\sum_{i i^{\prime}, \sigma, \sigma^{\prime}, m_{-}} \frac{V_{\alpha, i}^{*} V_{\alpha^{\prime}, i^{\prime}}}{E_{m_{-}}-E_{0}+\epsilon_{\alpha^{\prime}}} \gamma_{n, n^{\prime}}^{m_{-}}\left(i i^{\prime}, \sigma \sigma^{\prime}\right),
$$

where

$$
\begin{aligned}
& \gamma_{n, n^{\prime}}^{m_{+}}\left(i i^{\prime}, \sigma \sigma^{\prime}\right)=\left\langle n\left|d_{i \sigma}\right| m_{+}\right\rangle\left\langle m_{+}\left|d_{i^{\prime} \sigma^{\prime}}^{\dagger}\right| n^{\prime}\right\rangle, \\
& \gamma_{n, n^{\prime}}^{m_{-}}\left(i i^{\prime}, \sigma \sigma^{\prime}\right)=\left\langle n\left|d_{i \sigma^{\prime}}^{\dagger}\right| m_{-}\right\rangle\left\langle m_{-}\left|d_{i^{\prime} \sigma}\right| n^{\prime}\right\rangle .
\end{aligned}
$$

Equations (5)-(10) constitute the cornerstone of the formalism. The Hamiltonian $\mathcal{H}_{\text {cotun }}$ in Eq. (5) describes the scattering of a quasiparticle from the single-particle state $\alpha^{\prime}$ to $\alpha$ in the electrodes together with a transition between two many-body states of the MS within the $q_{0}$ manifold. Three types of elementary processes are described by the effective cotunneling Hamiltonian: elastic processes in which transport electrons are transferred between both electrodes without changes in the central region, the creation of an electron-hole pair in a given electrode with the corresponding transition in the central island, and inelastic tunneling events. In all of them, it is apparent from Eqs. (9) and (10) that the excitations within the $q_{0}$ manifold in the MS occur via virtual transitions to the 

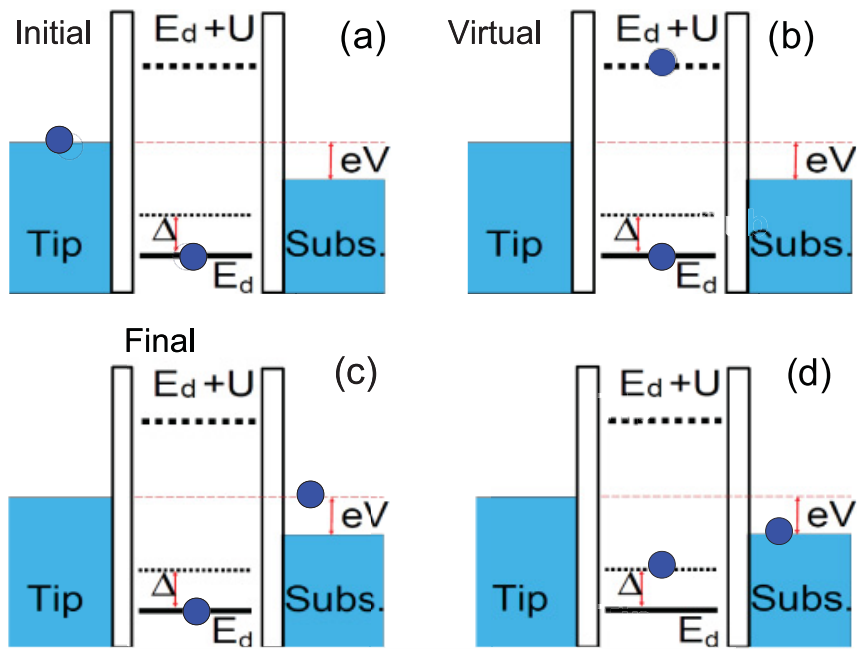

FIG. 1. (Color online) Scheme of the cotunneling transport process through a multiplet of orbital levels far from resonance. (a) Initial state, with the magnetic system in its ground state. (b) Virtual intermediate state, in which an electron is added to the magnetic system. (c) Final state corresponding to elastic transport process without change in the magnetic system. (d) Inelastic transport process with change of the magnetic system state.

charged manifolds $q=-$ and $q=+$. A scheme of some of these processes can be seen in Fig. 1 .

Very much like in the case of effective Kondo models, the quasiparticle scattering events can be classified into four groups depending on whether they include, or not, spin-flip and/or electrode transitions. In turn, the spin-conserving events are split in two more groups, depending on weather or not they have spin-dependent amplitudes. Because of the spin rotational invariance imposed in the tunneling Hamiltonian (3), quasiparticle spin-flip events imply spin transfer to the MS. Finally, the last term in Eq. (5) describes a renormalization of the many-body levels of the MS and can be re-adsorbed into a new Hamiltonian for the central part, $\mathcal{H}_{\mathrm{MS}}^{\prime}=\mathcal{H}_{\mathrm{MS}}+$ $\sum_{\alpha} \hat{\mathcal{O}}_{\alpha \alpha}^{(-)}$, so it will be omitted in the following analysis.

For a fixed set of initial and final quasiparticle states, $\alpha, \alpha^{\prime}$, the matrices (8) and (7) have, at most, the dimension of the $q_{0}$ manifold. For instance, as discussed in detail in Sec. III in the so-called Anderson model, when the states with $q=q_{0}$ in the island are those of an unpaired electron, the dimension of the matrices (7) and (8) is 2, corresponding to the two spin projections of a spin $1 / 2$. As a result, the Hamiltonian (5) describes a Kondo coupling between the electrode and the spin $1 / 2$ of the MS.

\section{B. Master equation, transition rates, and current}

The procedure described above yields an effective Hamiltonian of the MS coupled to the electrodes for which the states of the $q= \pm$ charge have been integrated out. The effective total Hamiltonian of the electrodes coupled to the $q_{0}$ manifold reads

$$
\mathcal{H}_{\text {eff }}=\sum_{n} E_{n}|n\rangle\langle n|+\mathcal{H}_{T}+\mathcal{H}_{S}+\mathcal{H}_{\text {cotun }} .
$$

This Hamiltonian serves as a starting point to calculate both current and dynamics of the many-body states of the MS within the $q_{0}$ manifold. For that matter, we take the conventional system plus reservoir approach ${ }^{31}$ where the system is defined by the many-body states of the $q_{0}$ charge state and the reservoir is given by the electrons in the electrodes. The system-reservoir coupling is described by $\mathcal{H}_{\text {cotun }}$, and is responsible for the dissipative dynamics of the $n$ states in the MS. This dynamics can be then described by a master equation for the reduced density matrix, after the degrees of freedom of the electrodes are integrated out. ${ }^{31}$ The time evolution of the populations of the MS states, $P_{n}$, is then given by a Markovian master equation

$$
\frac{d P_{n}}{d t}=\sum_{n^{\prime}} W_{n^{\prime}, n} P_{n}^{\prime}-P_{n} \sum_{n^{\prime}} W_{n, n^{\prime}},
$$

where the transition rates $W_{n n^{\prime}}$ for the MS to go from state $n$ to $n^{\prime}$ due to quasiparticle scattering in the electrodes are calculated by applying the Fermi golden rule with the perturbation given by the tunneling Hamiltonian (5). In deriving Eq. (12), it is necessary to assume that both the correlation time of the electrons in the leads, of the order of $\hbar /\left(k_{b} T\right)$, and the period $\sim \hbar /\left(E_{n}-E_{n^{\prime}}\right)$ of the system coherent evolution are much shorter than the scattering time, $\sim 1 / W_{n, n^{\prime}}{ }^{32}$ The steady state solutions of this master equation depend, in general, on the Hamiltonian parameters, the temperature, and the bias voltage. At zero bias, the steady state solutions are those of thermal equilibrium. At finite bias, $P_{n}(V)$ can depart significantly from equilibrium depending on the relative efficiency of the transport-assisted excitations and relaxations. ${ }^{22}$

The rates $W_{n, n^{\prime}}$ are the sum of scattering processes in which the initial and final electrode and spin quantum numbers of the quasiparticle are well defined,

$$
W_{n, n^{\prime}}=\sum_{\sigma \sigma^{\prime}, \eta \eta^{\prime}} W_{n, n^{\prime}}^{\eta \sigma, \eta^{\prime} \sigma^{\prime}} .
$$

An explicit expression for the spin- and electrode-dependent scattering rate $W_{n, n^{\prime}}^{\eta \sigma, \eta^{\prime} \sigma^{\prime}}$ is given in Appendix B, Eq. (B1). The expression involves a convolution over the energydependent density of states and effective cotunneling rates. A simpler expression is obtained by doing a number of approximations, ${ }^{27,28}$ as explained in Appendix B. First, we assume that the electrodes have a flat density of states within a bandwidth larger than all relevant energy scales in the problem: temperature, bias, and the excitations energies of the MS within the $q_{0}$ manifold. Second, we neglect the energy dependence of the hopping matrix elements $V_{k \eta, i}=V_{\eta, i}{ }^{33}$ These approximations are justified in IETS experiments where the temperature is at most a few kelvins and the applied bias is below $50 \mathrm{mV}$. If we introduce the excitation energy associated with the transition between $n^{\prime}$ and $n$ states in the $q_{0}$ manifold, $\Delta_{n n^{\prime}}=E_{n}-E_{n^{\prime}}$, and we define the average energy $\bar{\epsilon}_{n n^{\prime}}^{\eta \eta^{\prime}}=1 / 2\left(\mu_{\eta}+\mu_{\eta^{\prime}}+\Delta_{n n^{\prime}}\right)$, the transition rates $W_{n n^{\prime}}^{\eta \eta^{\prime}}$ obtained in Appendix B can be expressed as

$$
W_{n n^{\prime}}^{\eta \eta^{\prime}} \approx \sum_{\sigma \sigma^{\prime}} \frac{2 \pi \rho_{\eta \sigma} \rho_{\eta^{\prime} \sigma^{\prime}}}{\hbar} \mathcal{G}\left(\mu_{\eta}-\mu_{\eta^{\prime}}+\Delta_{n n^{\prime}}\right) \Sigma_{n n^{\prime}}^{\eta \sigma, \eta^{\prime} \sigma^{\prime}}\left(\bar{\epsilon}_{n n^{\prime}}^{\eta \eta^{\prime}}\right)
$$


where $\mathcal{G}(\omega)=\frac{\omega}{1-\exp [-\beta \omega]}$ and $\rho_{\eta \sigma}$ are the spin- and electroderesolved density of states. The many-body matrix elements $\Sigma_{n n^{\prime}}^{\eta \sigma, \eta^{\prime} \sigma^{\prime}}(\bar{\epsilon})$ are given by

$$
\Sigma_{n n^{\prime}}^{\eta \sigma, \eta^{\prime} \sigma^{\prime}}(\bar{\epsilon})=\left|\left\langle n\left|\left(\hat{\mathcal{O}}_{\bar{k} \eta \sigma, \bar{k}^{\prime} \eta^{\prime} \sigma^{\prime}}^{(+)}-\hat{\mathcal{O}}_{\bar{k}^{\prime} \eta^{\prime} \sigma^{\prime}, \bar{k} \eta \sigma}^{(-)}\right)\right| n^{\prime}\right\rangle\right|^{2},
$$

where $\bar{k} \equiv k(\bar{\epsilon})$, i.e., the quasiparticle energy that appears in the denominators, is replaced by the corresponding biasdependent average energy $\bar{\epsilon}_{n n^{\prime}}^{\eta \eta^{\prime}}$.

In this context, the current is given by ${ }^{18,22}$

$$
I_{T \rightarrow S}=e \sum_{n, n^{\prime}} P_{n}(V)\left(W_{n, n^{\prime}}^{S \rightarrow T}-W_{n, n^{\prime}}^{T \rightarrow S}\right),
$$

where $e$ is the (negative) electron charge. This equation has a physically transparent meaning: The current is proportional to the transition rates of quasiparticles changing electrodes. These rates involve transitions of the MS from the state $n$, which is occupied with probability $P_{n}(V)$, to state $n^{\prime}$, including elastic events $n=n^{\prime}$.

Our convention for the applied bias is such that $e V=$ $\mu_{S}-\mu_{T}$ (electrons move from tip to surface for a positive applied bias). In general, the bias voltage implies a small charge accumulation both in the tip and the surface which in turn involves a shift of their chemical potentials with respect to their equilibrium value, denoted by $E_{F}$. Since the capacitance of the surface is much larger than that of the tip, we assume that the bias shifts the chemical potential of the tip only, $\mu_{T}=E_{F}-e V$, and leaves the one of the surface-adatom system unchanged. As we show below, this assumption makes it possible to account for the conductance asymmetry reported experimentally. 8,10

In the following, we shall express the differential conductance in units of

$$
g_{0}=\frac{G_{0}}{2} \rho_{S} \rho_{T}\left(J_{T S}^{2}+\mathcal{W}_{T S}^{2}\right),
$$

where $G_{0}=2 e^{2} / h$ is the quantum of conductance, $\rho_{\eta}=$ $\sum_{\sigma} \rho_{\eta \sigma}$, and $J_{T S}$ and $\mathcal{W}_{T S}$ are simply the generalizations of the (momentum independent) exchange and direct coupling respectively, that appear in the Anderson model, as will be shown below:

$$
J_{T S}=2 V_{S}^{(M)} V_{T}^{(M)}\left[\frac{1}{\mu_{e}-E_{F}}+\frac{1}{E_{F}-\mu_{h}}\right]
$$

and

$$
\mathcal{W}_{T S}=\frac{V_{S}^{(M)} V_{T}^{(M)}}{2}\left[\frac{1}{\mu_{e}-E_{F}}-\frac{1}{E_{F}-\mu_{h}}\right],
$$

where $V_{\eta}^{(M)}$ is the largest coupling between electrode $\eta$ and the orbitals of the MS.

\section{Summary of the method}

The approach described above can be implemented in a wide range of situations following a sequence of well-defined steps:

(1) Diagonalization of the MS Hamiltonian in the 3 relevant charge states, $q=q_{0}-1, q_{0}, q_{0}+1$, providing $|q, n\rangle$ and $E_{q, n}$.
(2) Computation of the matrix elements (7) and (8) of the effective tunneling Hamiltonian operator, which requires the calculation of the many-body matrix elements $\gamma$ [Eqs. (9) and (10)] and the $\mathcal{O}$-matrix prefactors.

(3) Calculation of the scattering rates (14), which depend on bias, temperature, MS-electrode coupling, electrode density of states, and MS wave functions.

(4) Finding the nonequilibrium steady state solutions $P_{n}(V)$ of the master equation (12).

(5) Evaluation of the current using Eq. (16).

\section{Comparison with other cotunneling theories}

The calculation of cotunneling current has been widely studied before, using different methodologies, mainly in the context of quantum dots ${ }^{23,24,34}$ and, more recently, molecules. ${ }^{35-39}$ For instance, in Refs. 35-38 they compute the cotunneling scattering rates by truncating the $T$ matrix down to second order in the electrode coupling. On the other hand, a more formal and accurate treatment, valid also in the strong-coupling regime, was introduced in Ref. 39, where the nonequilibrium Keldysh Green's function formalism was used to study the inelastic spectroscopy of single adsorbed molecules.

Whereas the current obtained using these different methods is the same, our approach permits us to derive an effective Hamiltonian which, in the adequate limit, is the same as the effective Kondo Hamiltonian used extensively in previous works. ${ }^{14-22}$ An interesting work addressing the relation between the multiple-impurity Anderson model at half-filling and a Kondo model was presented in Ref. 40, where the authors proved that a Hubbard chain of $N$ impurities coupled in parallel can be described with a $S=N / 2$ SU(2) spin Kondo model. In Ref. 29, the authors used the same generalized Schrieffer-Wolff transformation to relate a singlet-triplet Anderson impurity with a spin model close to its quantum phase transition.

Our approach, based on an effective cotunneling Hamiltonian directly obtained from the exact description of the magnetic system, provides a microscopic justification of earlier phenomenological works, at the time that it keeps the simplicity that allows us to calculate the current as described above.

\section{SINGLE-ORBITAL ANDERSON MODEL}

In this section we revisit the very well known Anderson model $^{25}$ for which the MS is a single-site Hubbard model:

$$
\mathcal{H}_{\mathrm{MS}}=E_{d} \sum_{\sigma} d_{\sigma}^{\dagger} d_{\sigma}+U n_{\downarrow} n_{\uparrow},
$$

where $E_{d}$ is the on-site energy level, $U$ the on-site Coulomb repulsion, and $n_{i \sigma}=d_{i \sigma}^{\dagger} d_{i \sigma}$. We now derive an effective cotunneling Hamiltonian which, as we show below, turns out to be identical to the spin-1/2 Kondo model by means of a Schrieffer-Wolff transformation. ${ }^{25,26}$ By so doing, we test the validity of our approach and shed some light on the origin of the large contribution of the inelastic spin-assisted tunneling to the conductance.

The single-site Hubbard Hamiltonian has only 3 possible charge states, empty, singly, and doubly occupied. The singly 
occupied manifold has two states, $|\uparrow\rangle$ and $|\downarrow\rangle$ with energy $E_{d}$. The empty and doubly occupied manifolds have only 1 state each, $|\uparrow \downarrow\rangle$ with energy $2 E_{d}+U$ for the + manifold, and $|0\rangle$ with energy 0 for the - manifold, respectively. If $E_{d}+U \gg$ $E_{F} \gg E_{d} \gg k_{b} T$ the ground state has $q_{0}=1$ and classical charge fluctuations are frozen. Hence, the virtual transition operators acting on the $q_{0}=1$ space have dimension two and can be expressed as Pauli matrices, acting on the spin space.

After a straightforward calculation we find the effective cotunneling Hamiltonian with 3 contributions. First, the famous exchange-assisted Kondo term: ${ }^{25,26}$

$$
\mathcal{H}_{\mathrm{cot}, 1}=\sum_{k k^{\prime}, \eta \eta^{\prime}, \sigma \sigma^{\prime}} J_{k k^{\prime}, \eta \eta^{\prime}} \overrightarrow{\mathcal{S}} \cdot \frac{\vec{\tau}_{\sigma \sigma^{\prime}}}{2} f_{k \eta \sigma}^{\dagger} f_{k^{\prime} \eta^{\prime} \sigma^{\prime}}
$$

with

$$
J_{k k^{\prime}, \eta \eta^{\prime}}=2 V_{k \eta} V_{k^{\prime} \eta^{\prime}}^{*}\left[\frac{1}{E_{d}+U-\epsilon_{k \eta \sigma}}+\frac{1}{\epsilon_{k^{\prime} \eta^{\prime} \sigma^{\prime}}-E_{d}}\right] \text {. }
$$

The second term in the Hamiltonian corresponds to a direct (spin-independent) interaction, also obtained in the SchriefferWolff transformation, 25

$$
\mathcal{H}_{\mathrm{cot}, 2}=\sum_{k k^{\prime}, \eta \eta^{\prime}, \sigma} 2 \mathcal{W}_{k k^{\prime}, \eta \eta^{\prime}} f_{k \eta \sigma}^{\dagger} f_{k^{\prime} \eta^{\prime} \sigma}
$$

where

$\mathcal{W}_{k k^{\prime}, \eta \eta^{\prime}}=\frac{V_{k \eta} V_{k^{\prime} \eta^{\prime}}^{*}}{2}\left[\frac{1}{E_{d}+U-\epsilon_{k \eta \sigma}}-\frac{1}{\epsilon_{k^{\prime} \eta^{\prime} \sigma^{\prime}}-E_{d}}\right]$.

Notice how in this model, the exchange-assisted $J_{k k^{\prime}, \eta \eta^{\prime}}$ and the direct tunneling term $\mathcal{W}_{k k^{\prime}, \eta \eta^{\prime}}$ have a common origin, namely, virtual charging of the magnetic site. Importantly, we see how we can have the spin-flip term much larger than the direct term. In particular, in the so-called symmetric case, for which $E_{d}+U-E_{F}=E_{F}-E_{d}$, the direct term vanishes altogether, due to a cancellation between the electron addition and hole addition channels. In that situation only the spin-flip assisted tunneling would be possible. Thus, the cotunneling picture provides a natural scenario for the large contribution of the inelastic contribution to the conductance. This scenario differs from the perturbative derivation of a spin exchange Hamiltonian used in Refs. 19,41-43, where the exchange tunneling was assumed to be small compared to the spin-conserving channel. Finally, a third term $\mathcal{H}_{3}=$ $\sum_{k, \eta, \sigma} \mathcal{H}_{3}(k, \eta, \sigma)$ is obtained, which can be considered as a renormalization of the on-site energy level.

\section{STACKS OF CoPc MOLECULES}

In this section we model the IETS experiments of stacks of cobalt phthalocyanine molecules ( $\mathrm{CoPc}$ ) deposited on $\mathrm{Pb}(111){ }^{8,10}$ CoPc molecules are planar molecules with $D_{4 h}$ symmetry and a single cobalt (Co) atom at its center, surrounded by four nitrogen neighbors and enclosed by aromatic macrocycles. A single $\mathrm{CoPc}$ has a ground state with spin $S=1 / 2$, corresponding to an unpaired electron presumably in the $d_{z^{2}-r^{2}}$ orbital of Co. In a stack with $N+1$ CoPc molecules, the $\mathrm{CoPc}$ in contact with the $\mathrm{Pb}$ surface acts as a dead layer that isolates the remaining $N$ molecules.

The stacking seems to be such that cobalt atoms are underneath nitrogen atoms of the adjacent molecule. The IETS results ${ }^{8,10}$ of stacks with $N$ active $\operatorname{CoPc}(N+1$ molecules in total) can be interpreted as if the molecules are coupled via an antiferromagnetic coupling, which presumably comes from superexchange between two cobalt atoms coupled to a common nitrogen atom. The observed spin-flip excitations were successfully described using a Heisenberg model with an antiferromagnetic (AF) coupling $J \simeq 18 \mathrm{meV}$.

Whereas the Heisenberg model accounts for the observed excitation energies, it cannot account for either the transport mechanism or the fact that the conductance in this system is very asymmetric. In particular, some inelastic steps seen at a given bias polarity are not seen when the bias sign is reversed. Additional experiments where the charge state of the molecular stack was controlled using the STM tip as a local gate make it necessary to go beyond spin-only models. ${ }^{10}$ The observed excitation energies could be accounted for using a Hubbard model, rather than a Heisenberg model:

$$
\begin{aligned}
\mathcal{H}_{\mathrm{MS}}= & E_{d} \sum_{i \sigma} d_{i \sigma}^{\dagger} d_{i \sigma}+U \sum_{i} n_{i \downarrow} n_{i \uparrow} \\
& +t \sum_{i, \sigma}\left(d_{i \sigma}^{\dagger} d_{i+1 \sigma}+\text { H.c. }\right) .
\end{aligned}
$$

Here $E_{d}$ stands for the energy of the $d_{z^{2}-r^{2}}$ orbital with respect to the Fermi energy, which we take at $0, U$ stands for the on-site Coulomb repulsion, and $t$ for the Co-Co hopping, which actually occurs through the common nitrogen neighbor. In the strongly insulating limit, $U \gg t$ and at half-filling (1 unpaired electron per cobalt atom), the Hubbard model has the same low-energy excitation spectra as the Heisenberg model with $J=\frac{4 t^{2}}{U}$. Away from half-filling, when the molecular stack is charged, the mapping to the Heisenberg model is no longer possible but still the excitation energies observed experimentally are accounted for by the Hubbard model. ${ }^{10}$ Here we focus on the half-filling case and we apply our formalism to short Hubbard chains with $N=2,3,4$ sites. We take $U=1.5 \mathrm{eV}$ which imposes $t=82 \mathrm{meV}(J \approx 18 \mathrm{meV})$, in accordance with the experimentally observed value. ${ }^{8,10}$

\section{A. The dimer}

The eigenvalues and eigenvectors of the Hubbard dimer can be found analytically, both for the half-filling state and the two states with 1 and 3 electrons. At half-filling $\left(q=q_{0}\right)$ the ground state corresponds to a spin singlet, $S=0$, while the first excited state corresponds to a spin triplet, $S=1$ with excitation energy $J$ neglecting terms of order $t^{4} / U^{3}$, see Fig. 2(b) (in agreement with the experimental results ${ }^{8,10}$ ). The electron addition and hole addition energies are $E_{d}+U$ and $-E_{d}$, respectively. Thus, for the MS to be at half-filling we must have $-U<E_{d}<0$. The states with charge $q= \pm$ correspond to those of a single electron and a single hole, respectively.

As evinced by Eqs. (7) and (8), cotunneling can take place through virtual hole and virtual electron addition $\left(q_{ \pm}\right.$channels). These two processes are weighted by an energy factor $1 /\left(\mu_{e, h}-E_{F}\right)$, which explicitly depend on the on-site energy $E_{d}$ and electrode Fermi level $E_{F}$. Therefore, by changing the on-site energy with respect to the Fermi level we can go from an electron-mediated cotunneling $\left(\left|\mu_{e}-E_{F}\right| \ll\left|\mu_{h}-E_{F}\right|\right)$ 


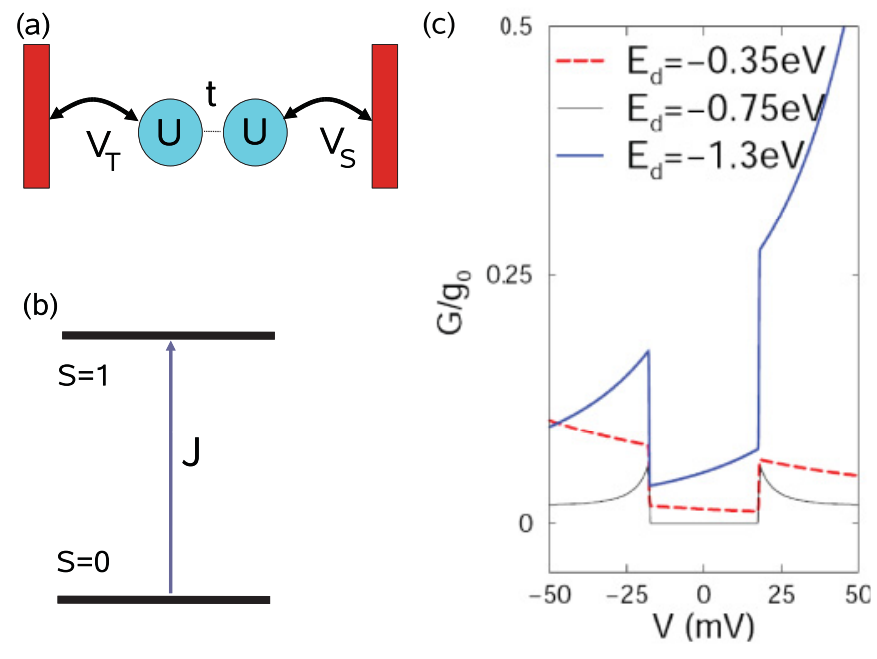

FIG. 2. (Color online) (a) Scheme of a two-site Hubbard dimer connected to two electrodes. (b) Lowest energy levels of Hubbard dimer at half-filling in terms of the exchange constant $J$. (c) $d I / d V$ as a function of applied bias. Here $\rho_{S} V_{S}=5, \rho_{T} V_{T}=1$, and $T=0.4 \mathrm{~K}$.

to a hole-mediated cotunneling $\left(\left|\mu_{e}-E_{F}\right| \gg\left|\mu_{h}-E_{F}\right|\right)$, going through the electron-hole symmetry point (EHSP) where both contributions are equal. Figure 2(c) shows the calculated $d I / d V$ spectra obtained for the Hubbard dimer for three different values of the on-site energy $E_{d}: E_{d}=-U / 2$, the EHSP, $E_{d}=-0.35 \mathrm{eV}$, for which virtual transitions to the $q_{-}$ manifold are favored, and $E_{d}=-1.3 \mathrm{eV}$, which favors virtual transitions to the $q_{+}$manifold. Whereas the excitation step at $\pm e V=J$ is present in all of them, both the magnitude and the bias dependence of the elastic contribution depend critically on $E_{d}$. At the EHSP, the elastic conductance is zero, as in the Anderson model, and the nonmonotonic line shape right above the inelastic step is due to the depletion of the occupation of the ground state in favor of the excited state, a nonequilibrium effect discussed in our previous work. ${ }^{22}$

Both the elastic and the inelastic contributions increase when $E_{d}$ is taken away from the EHSP. When $E_{d}=-1.3 \mathrm{eV}$, the virtual transition to the $q_{+}$manifold is dominant and cotunneling is mediated by the addition of an electron. As we mentioned in Sec. II B, our bias convention is such that positive bias $V$ results in an increment of the tip chemical potential with respect to the molecules and the surface. Thus, for $V>0$ it becomes easier to add an electron to the system, increasing the global conductance. For $V<0$, instead, the chemical potential of the tip is decreased, making it relatively harder to charge the dimer with an electron and reducing the cotunneling conductance thereby. The large conductance observed at positive bias corresponds to the tail of a resonance in the transmission above the Fermi energy, which is probably associated with resonant tunneling through a molecular level. In the case of $E_{d}=-0.35 \mathrm{eV}$ the situation is reversed. The virtual transition to the $q_{-}$manifold is dominant; i.e., cotunneling is mediated by the addition of a hole (or the removal of an electron). In this case a positive bias makes it harder for the electron to tunnel out of the system, decreasing the conductance. Thus, in our calculation the asymmetry of the conductance comes from the assumption that the bias shifts mostly the tip chemical potential, and not the surface, and the fact that one of the two cotunneling channels (virtual addition of either an electron or a hole) is dominant. Comparing with the experimental results, ${ }^{8}$ we infer that the double $\mathrm{CoPc}$ molecule system is close to the electron addition point. This has been further confirmed by additional experiments by the same group. ${ }^{10}$

\section{B. The trimer and the tetramer}

We now consider the Hubbard chains with either $N=3$ or $N=4$ sites and try to model the CoPc molecular stacks with 3 and 4 active molecules, respectively. ${ }^{8}$ We assume that $t$ and $U$ take the same values as before and that there is one electron per site in the ground state. We label the sites from $n=1$ to $n=N$, starting from the molecule closest to the tip. For $N=3$, the ground state and first excited state have $S=1 / 2$ and the second excited state has $S=3 / 2$ (see Fig. 3). Thus, we expect two inelastic transitions, at energies $J$ and $3 J / 2$. For the $N=4$ chain, the ground state has $S=0$ and the two lowest energy excited states, both with $S=1$, have excitation energies $0.7 J$ and $1.4 J$, see Fig. 4 . Again, two inelastic steps are expected at those energies.

In Figs. 3(a) and 4(a) we show the conductance for $N=3$ and $N=4$, respectively, assuming that the electrons can tunnel from the tip to the $n=1$ site only and from the $n=N$ site to the surface only. As in the case of the dimer, we take 3 different values for $E_{d}$ : hole mediated, electron mediated, and EHSP. On top of the symmetry trends already discussed for the dimer, we see how in the EHSP only the lowest energy transition is seen both in the $N=3$ and $N=4$ cases. This suggests that not only the elastic contribution vanishes, as in the case of the Anderson model and the Hubbard dimer, but also some of the inelastic transitions can be suppressed possibly due to the destructive interference between the hole and electron channels.

In the case of Figs. 3(a) and 4(a), where only the sites at the end of the chain are coupled to either the tip or the surface, the steps are visible for both signs of $V$, at odds with the experimental observations. ${ }^{8}$ In an attempt to explore a scenario in which the height of the steps are only visible at a given polarity, we have considered a situation where electrons can tunnel from the tip to sites other than $n=1$ and from the surface to sites other than $n=N$. By so doing, we can obtain $d I / d V$ curves where the steps are depleted for $V<0$ [Figs. 3(b) and 4(b)]. However, we think that a more plausible explanation would come from a microscopic calculation including more than 1 orbital per molecule. It must also be mentioned the broadening of the excitations observed experimentally is larger than $5.4 k_{B} T$, which indicates than neglecting the intrinsic broadening due to the coupling to the continuum of states of the electrodes is not fully justified. ${ }^{44}$

\section{MAGNETIC ADATOMS}

We now consider spin IETS through a single Mn atom deposited on a $\mathrm{Cu}_{2} \mathrm{~N}$ surface. This system has been widely studied experimentally ${ }^{3,4,6}$ and theoretically, ${ }^{15-22,45,46}$ in most instances modeling the Mn spin with an effective spin model. Here we go beyond the spin model picture and we use a 

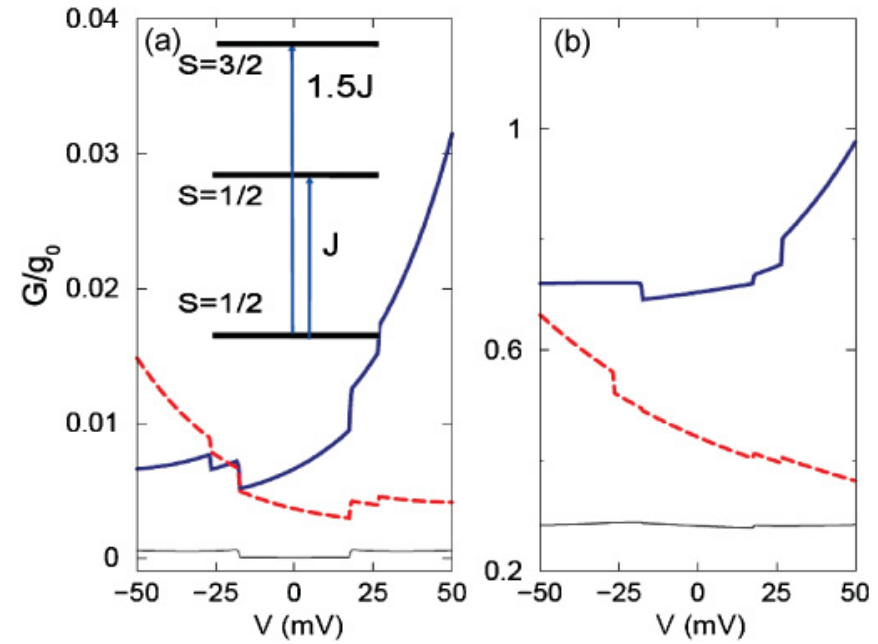

FIG. 3. (Color online) $d I / d V$ as a function of applied bias for $N=3$ with on-site energy $E_{d}=-0.35 \mathrm{eV}$ (red-dashed line), $E_{d}=$ $-0.75 \mathrm{eV}$ (thin-black line), and $E_{d}=-1.2 \mathrm{eV}$ (thick-blue line). (a) Serial Hubbard trimer with $V_{S, i}=2 \delta_{i, 3}$ and $V_{T, i}=5 \delta_{i, 1}$. (b) Multiple electrode connected Hubbard trimer with with $\rho_{S} \mathbf{V}_{\mathbf{S}}=(2,1,1)$ and $\rho_{S} \mathbf{V}_{\mathbf{S}}=(2,3,5)$. Inset: Scheme of the lowest energy levels. The other parameters are kept as in Fig. 1.

multiorbital Anderson Hamiltonian for the $5 d$ electrons of the $\mathrm{Mn}^{+2}$ ion which includes Coulomb interaction, spinorbit coupling, and crystal field. Transport occurs via virtual transitions to the many-body states with either 4 or $6 d$ electrons. Our approach requires the exact diagonalization of the fermionic model in the 3 relevant charge states, with 4, 5, and 6 electrons. Below we describe the multiorbital Anderson model and the transport calculation, and compare with the experimental results in Ref. 4.
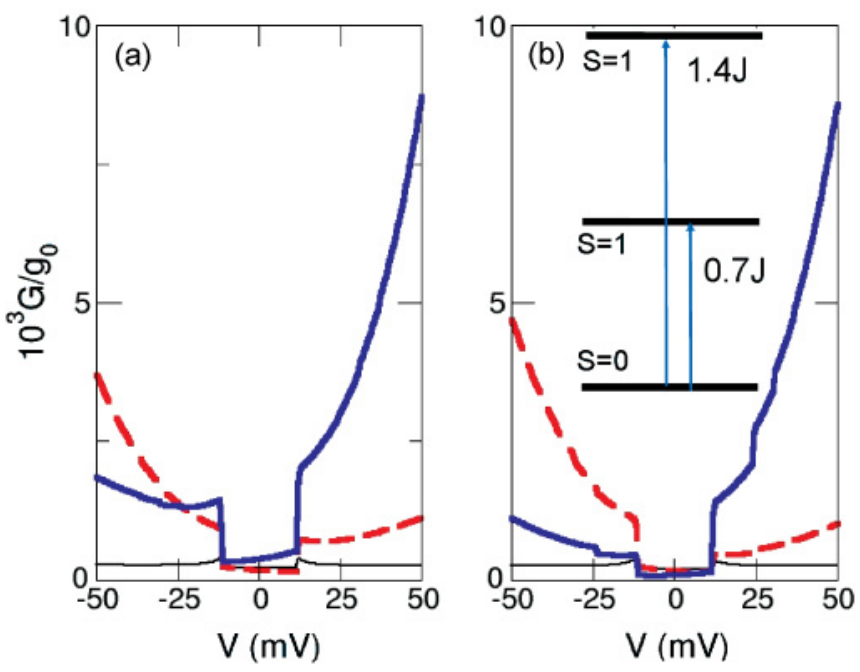

FIG. 4. (Color online) $d I / d V$ as a function of applied bias for $N=4$ with on-site energy $E_{d}=-0.35 \mathrm{eV}$ (red-dashed line), $E_{d}=$ $-0.75 \mathrm{eV}$ (thin-black line), and $E_{d}=-1.2 \mathrm{eV}$ (thick-blue line). (a) Serial Hubbard tetramer with $V_{S, i}=3 \delta_{i, 4}$ and $V_{T, i}=10 \delta_{i, 1}$. (b) Multiple electrode connected Hubbard tetramer with with $\rho_{S} \mathbf{V}_{\mathbf{S}}=$ $(0,0,0.2,10)$ and $\rho_{t} \mathbf{V}_{\mathbf{S}}=(3,0.3,0,0)$. Inset: Scheme of the lowest energy levels. The other parameters are kept as in Fig. 1.

\section{A. Magnetic system Hamiltonian}

Here we describe our model Hamiltonian for the Mn ion in the $\mathrm{Cu}_{2} \mathrm{~N}$ surface. The purpose of our model is to provide a minimal fermionic Hamiltonian that accounts for the data, rather than to provide a realistic description of the $\mathrm{Mn}$ ion on the surface. Density functional calculations $s^{4,47,48}$ suggest that the $\mathrm{Mn}$ adatom transfers charge to the $\mathrm{CuN}$ surface and creates bonds with its neighboring $\mathrm{N}$ atoms. As a result, the $\mathrm{Mn}$ adatom becomes a $\mathrm{Mn}^{2+}$ ion that has lost its two $4 s$ electrons. We model this system considering only the $3 d^{5}$ electrons of the Mn, including the electrostatic potential of the neighboring atoms. The Hamiltonian of the MS can be written as

$$
\mathcal{H}_{C}=H_{e e}+H_{\mathrm{CF}}+H_{\mathrm{SO}}+H_{\text {Zeem }},
$$

where $H_{e e}$ is the Coulomb repulsion between the $3 d$ electrons, $H_{\mathrm{CF}}$ is the crystal field Hamiltonian, $H_{\mathrm{SO}}$ is the spin-orbit Hamiltonian, and $H_{\text {Zeem }}$ is the Zeeman Hamiltonian associated to an applied magnetic field $\vec{B}$. The Coulomb matrix elements of the atomic orbitals can be expressed in terms of radial integrals which depend on the specific form of the approximate wave function and an angular part that can be obtained analytically. ${ }^{49}$ We have taken them from a calculation for an isolated ion using the GAUSSIAN package, ${ }^{50}$ which yields to the unscreened on-site Coulomb repulsion $U \simeq 24 \mathrm{eV}$, in accordance with unscreened Hartree-Fock calculations. ${ }^{51}$ Since screening in the real system makes $U$ much smaller than the single-ion calculation, we have downscaled the Coulomb matrix elements with an overall dielectric constant of $\varepsilon=4.7$ in order to obtain $U$ in the range of $5 \mathrm{eV}^{52}$

The energy $E_{d}$ of the $d$ levels before crystal splitting is included is kept as a free parameter in our theory. The crystal field term $H_{\mathrm{CF}}$ is built using a point charge model for the first $\mathrm{N}$ and $\mathrm{Cu}$ neighbors, ${ }^{53}$ whereas an effective dielectric constant $\varepsilon^{\prime}$ was introduced to account for the screening of the bare crystal field and fit the many-body spectrum to that of the single-ion Hamiltonian. ${ }^{54}$ Figure 5(a) shows the splitting of the five $d^{5}$ energy levels due to the crystal field, together with its dominant orbital contribution. Finally, the spin-orbit Hamiltonian reads

$$
H_{\mathrm{SO}}=\frac{\lambda}{\varepsilon^{\prime \prime}} \sum_{m, m^{\prime}, \sigma, \sigma^{\prime}}\left\langle m \sigma|\vec{L} \cdot \vec{S}| m^{\prime} \sigma^{\prime}\right\rangle d_{m \sigma}^{\dagger} d_{m^{\prime}, \sigma^{\prime}}
$$

where $\lambda=43 \mathrm{meV}$ corresponds to the value of the bare $\mathrm{Mn}^{2+}$ ion $^{54}$ and $\varepsilon^{\prime \prime}$ is another free parameter in our model.

The Hamiltonian (26) corresponding to the $3 d^{5}$ electrons was then diagonalized in the space of the 252 possible configurations, using the configuration interaction (CI) method. Analogously, the eigenvalues and eigenvectors of the $3 d^{4}$ and $3 d^{6}$ configurations were calculated in order to get the transition rates [Eq. (B1)]. The condition of stable configuration with $N_{e}=5$ electrons requires that $E_{G}(5) \leqslant E_{G}(4), E_{G}(6)$. In our case, this bound translates into the inequality $-24.1 \mathrm{eV}<$ $E_{d}<-18.9 \mathrm{eV}$. In particular, we choose $E_{d}$ in the middle of this energy window and, as will be shown in next section, results do not change significantly with $E_{d}$.

\section{B. $\mathrm{Mn}^{2+}$ energy spectra}

According to first Hund's rule, we expect that the spin of the ground state for the half-filled $d$ shell is $S=5 / 2$, which 
(a)
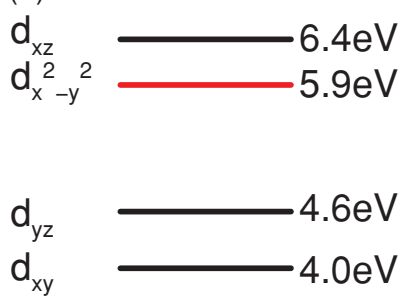

$d_{3 z^{2}-r^{2}}$

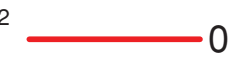

(b)

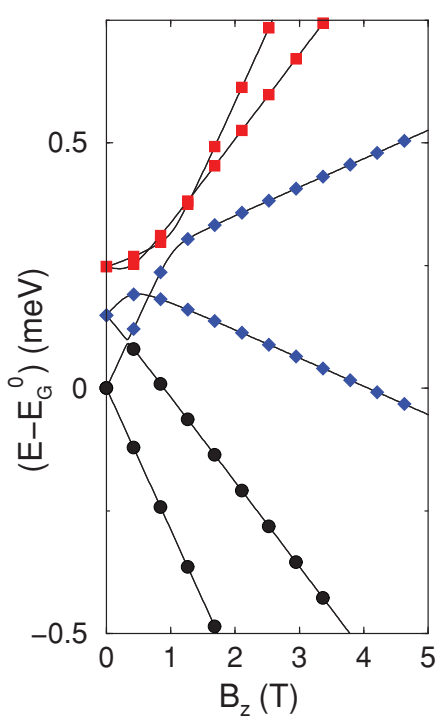

FIG. 5. (Color online) (a) Single-particle energy levels of the crystal field Hamiltonian with the dominant orbital contribution (only red levels are coupled by the crystal field). (b) Lowest energy spectra of the total Hamiltonian $\mathcal{H}_{\mathrm{MS}}$ for the $\mathrm{Mn}^{2+}$ ion on a $\mathrm{Cu}_{2} \mathrm{~N}$ surface as studied in Ref. 4. The dot symbols corresponds to the solution of the phenomenological spin model with $D=-0.39 \mathrm{meV}$ and $E=$ $0.06 \mathrm{meV}$. Here $E_{d}=-21.5 \mathrm{eV}, \varepsilon^{\prime}=11.3$, and $\varepsilon^{\prime \prime}=1.9$.

is what we obtain from the diagonalization of the model. The sixfold degeneracy at zero field is broken by the combined action of spin-orbit and crystal field. Due to the spin-orbit coupling, the total spin $S$ and total angular momentum $L$ are no longer good quantum numbers. However, our CI method allows us to calculate any of these expectations values. We have verified that for our calculation for the $\mathrm{Mn}^{2+},\langle S\rangle \approx 5 / 2$, while $\langle L\rangle \approx 0$, with a deviation smaller than $0.1 \%$. In the same way, $S_{z}$ is almost a good quantum number.

The location of the first neighbors of $\mathrm{Mn}$ is taken from Ref. 4. The values of $\varepsilon^{\prime}$ and $\varepsilon^{\prime \prime}$ are taken so that the lowest energy levels of the energy spectra obtained from the diagonalization of Eq. (26) are in agreement with those of the single-ion Hamiltonian, as shown in the Fig. 5(b). At zero field, the lowest energy doublet corresponds to $\left\langle S_{z}\right\rangle \approx \pm 5 / 2$. For the two pairs of excited levels, we get $\left\langle S_{z}\right\rangle \approx \pm 3 / 2$ and $\left\langle S_{z}\right\rangle \approx \pm 1 / 2$, in order of increasing energy. Figure 5(b) shows the magnetic field dependence of the low-energy spectra of the $\mathrm{Mn}^{2+}$ obtained using the CI calculation, together with the fitting to a phenomenological spin model $\mathrm{l}^{2-6,9,55}$

$$
\mathcal{H}_{S}=D S_{z}^{2}+E\left(S_{x}^{2}-S_{y}^{2}\right)+g \mu_{B} \vec{B} \cdot \vec{S} .
$$

The first two terms in Eq. (28) describe the single-ion magnetocrystalline anisotropy while the last one corresponds to the Zeeman splitting term under an applied magnetic field $\vec{B}$. The main magnetization direction $z$ in Eq. (28) depends on the substrate and magnetic atom nature. In the case of the $\mathrm{Mn}$ on a $\mathrm{Cu}_{2} \mathrm{~N}$ substrate, the $z$ axis is perpendicular to the surface. This result is also reproduced by our model (26).
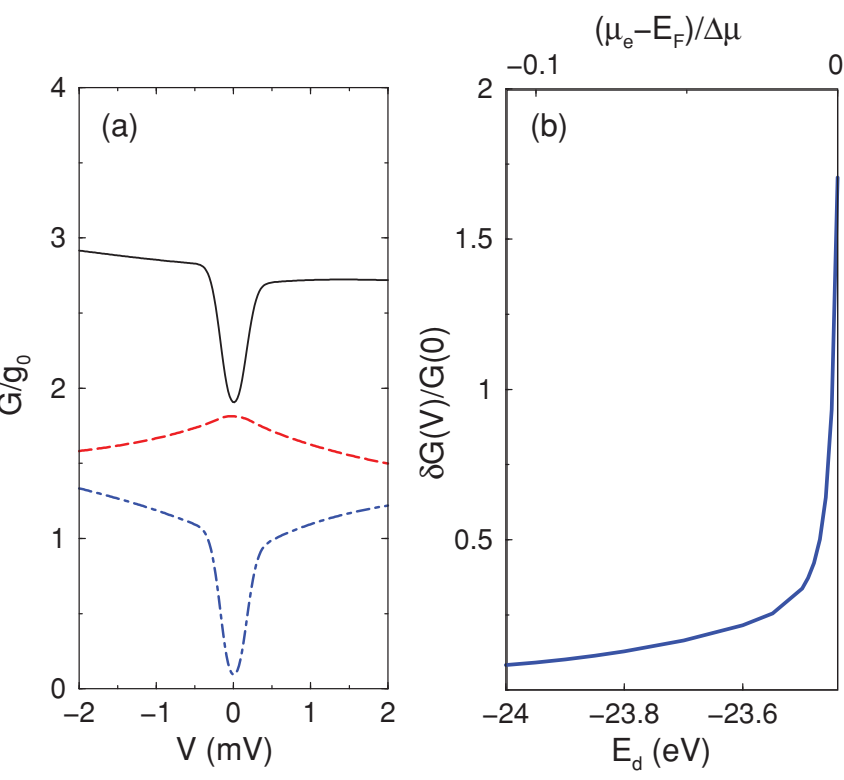

FIG. 6. (Color online) (a) Total (solid line), elastic (dashed line), and inelastic (dot-dashed line) $d I / d V$ as a function of applied bias for the $\mathrm{Mn}^{2+}$ ion $\left(\rho_{S} V_{S}=1, \rho_{T} V_{T, 3 z^{2}-r^{2}}=1, T=0.4 \mathrm{~K}\right.$, and $E_{d}=$ $-21.5 \mathrm{eV}$ ). (b) Ratio $\delta G(V) / G(0)$ for $V=2 \mathrm{meV}$ versus on-site energy $E_{d}$ (lower axis) or $\left(\mu_{e}-E_{F}\right) / \Delta \mu$ (upper axis), with $\Delta \mu=$ $\mu_{e}-\mu_{h}$. Other parameters as in Fig. 5.

\section{Transport}

Once the many-body eigenstates of Hamiltonian (26) are obtained, we are in position to study transport through the magnetic atom. For that matter, we need to specify the coupling of the $5 d$ orbitals to the tip and the substrate. As the $d I / d V$ spectra were recorded with the tip located exactly over the $\mathrm{Mn}$ atom, we will assume that the tip-atom tunneling is dominated by tunneling between the tip apex $s$ orbital and the $d_{3 z^{2}-r^{2}},{ }^{56}$ oriented along the adatom-tip axis. This assumption is at odds with existing DFT calculation, ${ }^{46}$ which shows that the tunneling through the Mn takes place mainly through the $s p$ orbitals of the $\mathrm{Mn}+$ substrate system, but it will still capture the main features. For the coupling with the substrate, the situation is significantly more complicated and we couple equally all the $d$ orbitals to the substrate; i.e., $V_{S, i}=V_{S}$. Another important parameter to properly account for the transport properties of the system is the Fermi level of the electrodes. Here we have assumed that the Fermi level of the $\mathrm{Cu}$ substrate coincides with its bulk Fermi level, $E_{F}=-4.5 \mathrm{eV}$, measured with respect to the vacuum level $(-7 \mathrm{eV}$ with respect to the bottom of the conduction band). ${ }^{57}$

The resulting $d I / d V$ is plotted in Fig. 6(a), where the elastic, inelastic, and total differential conductance are plotted. Our calculation reproduces both the line shape of the $d I / d V$ curves as well as the the relative contribution between the elastic and inelastic parts, $G_{\text {inel }} / G_{\text {el }} \simeq 0.5^{4,6}$ Within the model this ratio depends on the position of the charging energies of the atom, $\mu_{e}$ and $\mu_{h}$, with respect to the chemical potential of the electrodes. In Fig. 6(b) we show ratio $\delta G(V) / G(0)$, with $\delta G(V)=G(V)-G(0)$, as a function of the on-site energy level $E_{d}$ in the window of energies where the system ground state contains 5 electrons. As observed, the ratio $\delta G(V) / G(0)$ 
varies smoothly between 0.1 and 0.5 far from the resonant condition $\left|\mu_{h}-\mu_{\eta}\right|=0$. Closer to the resonance, the ratio $\delta G(V) / G(0)$ grows and it diverges as $\left|\mu_{h}-\mu_{\eta}\right| /\left(k_{b} T\right) \rightarrow 0$ where the cotunneling approach fails (not shown in the figure). Thus, our model yields a large inelastic signal, consistent with the experiments, without fine-tuning the on-site energy $E_{d}$. Notice that in the case of $\mathrm{Mn}$ on $\mathrm{Cu}_{2} \mathrm{~N}$ at $T=0.4 \mathrm{~K}$, the thermal broadening of the inelastic step is such that the inelastic conductance is nonzero even at zero bias.

\section{DISCUSSION AND CONCLUSIONS}

We have shown that the inelastic tunneling spectroscopy widely used to study magnetic molecules and atoms adsorbed on surfaces can be understood in terms of cotunneling. ${ }^{23,24}$ As the electrons go from the tip to the surface, the magnetic system must undergo virtual transitions to states with an extra electron or an extra hole. This picture holds both for elastic tunneling, in which case the MS returns to the original state after the virtual charging process, and inelastic tunneling, for which the states before and after the virtual charging are different. Thus, the origin of both elastic and inelastic conductance is the same, which accounts for the large inelastic signal reported experimentally in a variety of systems, including $\mathrm{Mn}, \mathrm{Fe}$, and $\mathrm{Co}$ on $\mathrm{Cu}_{2} \mathrm{~N}^{4}$ or $\mathrm{Fe}$ on $\mathrm{InSb}{ }^{12}$ Further support to this claim comes from comparison of the evolution of the $d I / d V$ as a function of an applied magnetic field of a quantum dot with a single resident electron in the Coulomb blockade regime ${ }^{58}$ and a single cobalt atom on $\mathrm{Cu}_{2} \mathrm{~N}$, both undergoing a transition from the Kondo regime at low field to inelastic steps at high field. Both systems show very similar $d I / d V$. In addition, our microscopic theory provides a natural starting point to describe both the appearance of Kondo correlations and their relation to the inelastic spin flips in the context of magnetic adatoms and molecules.

Our approach is based on the derivation of an effective cotunneling Hamiltonian acting only in the space of neutral configurations of the MS. The calculation of the effective Hamiltonian requires the exact diagonalization of the MS in the neutral subspace as well as the subspaces with one extra electron and one extra hole. From the formal point of view our results are in agreement with previous works based on a truncation of the $T$ matrix to second order in the coupling Hamiltonian. ${ }^{35-38}$ Our approach permits us to obtain an effective cotunneling Hamiltonian that can be compared with effective Kondo-like Hamiltonians proposed in most theoretical analysis of IETS experiments. ${ }^{14-22}$

We have also explored the origin of the experimentally observed asymmetry with respect to bias inversion in the $d I / d V$ curves. ${ }^{3,4,6-10}$ It comes from a combination of two ingredients. First, we need to consider that the bias voltage results in a shift of the chemical potential in the tip, the one in the surface remaining constant. Second, the energy level alignment of the MS must be such that one of the cotunneling channels, either virtual electron addition or virtual hole addition, is dominant.

In summary, we propose a method to describe single-spin inelastic electron tunneling spectroscopy which does not rely on effective spin models to describe both the magnetic system and the spin-flip assisted tunneling. Our approach provides a natural explanation for the large inelastic signals observed experimentally, and a microscopic mechanism for the spinassisted tunneling.

\section{ACKNOWLEDGMENTS}

We acknowledge fruitful discussions with J. J. Palacios, M. S. Santacruz, N. Lorente, C. F. Hirjibehedin, C. M. Canali, and C. Untiedt. This work was supported by MEC-Spain (MAT0767845, FIS2010-21883-C02-01, Grants JCI-2008-01885 and CONSOLIDER CSD2007-00010) and Generalitat Valenciana (ACOMP/2010/070).

\section{APPENDIX A: EFFECTIVE TUNNELING HAMILTONIAN}

We now use Eq. (4) to derive an effective Hamiltonian which acts on the reservoir fermions and on the $q_{0}$ subspace of the central island only. By so doing, we shall eliminate the $d^{\dagger}$ and $d$ operators from the effective Hamiltonian and, more importantly, we shall obtain a tunneling Hamiltonian for which the current can be derived straightforwardly. The matrix element between any two states in the $q_{0}$ manifold can be written as

$$
\left\langle N\left|\hat{\mathcal{V}}_{\text {tun }}\right| N^{\prime}\right\rangle=\left\langle\Psi_{f}(0)\left|\left\langle n\left|\hat{\mathcal{V}}_{\text {tun }}\right| \Psi_{f^{\prime}}(0)\right\rangle\right| n^{\prime}\right\rangle
$$

where $|N\rangle \equiv|n\rangle \otimes\left|\Psi_{f}(0)\right\rangle$, with $\left|\Psi_{f}\right\rangle$ a multielectronic Slater state describing independent Fermi seas of left and right electrodes. Importantly, the unperturbed states are product states of the left and right electrodes and the central island. These states can describe both the ground state of the MS with no excitations in the electrodes and excited states with an electron-hole pair in the electrodes and an excited state $n^{\prime}$ in the central island. Notice that the electron-hole pair can be either in one electrode or split in the left and right electrodes. In the second case, this excitation contributes to the net current flow. Now we need to evaluate matrix elements like

$$
\begin{aligned}
\left\langle\Psi_{f}(0)\right|\left\langle n\left|\mathcal{V}^{+}\right| M_{-}\right\rangle= & \sum_{\alpha, \mathbf{i}} V_{\alpha, \mathbf{i}}^{*}\left\langle\Psi_{f}(0)\left|f_{\alpha}\right| \Psi_{m f}(-)\right\rangle \\
& \times\left\langle n\left|d_{\mathbf{i}}^{\dagger}\right| m_{-}\right\rangle .
\end{aligned}
$$

Before going further, it is convenient to write down the explicit form of the electrode wave functions. If we denote the ground state of the electrodes in the Fermi sea with no excitations and in its neutral charge state as $|0\rangle$, we can write $\left|\Psi_{f}(0)\right\rangle \equiv f_{\alpha}^{\dagger} f_{\alpha}|0\rangle$, where we are creating an electronhole pair with quantum number $\alpha$. For the states with one electron excess (defect) we will have $\left|\Psi_{m f}(-)\right\rangle=f_{\beta}^{\dagger} f_{\alpha}^{\dagger} f_{\alpha}|0\rangle$ $\left(\left|\Psi_{m f}(+)\right\rangle=f_{\beta} f_{\alpha}^{\dagger} f_{\alpha}|0\rangle\right)$. The matrix element of the electrode operator in Eq. (A2) selects one and only one term in the electrode part of the sums $\sum_{M_{-}}=\sum_{m-} \sum_{m f}$. The term in question is such that

$$
\left|\Psi_{m f}(-)\right\rangle=f_{\gamma}^{\dagger}\left|\Psi_{f}(0)\right\rangle
$$

This relation is equivalent to writing $\left\langle\Psi_{f}(0)\left|f_{\gamma}\right| \Psi_{m f}(-)\right\rangle=$ $\left(1-n_{f}(\gamma)\right) \delta_{\beta \gamma}$, where $n_{f}(\gamma)=\left\langle\psi_{f}(0)\left|f_{\gamma}^{\dagger} f_{\gamma}\right| \psi_{f}(0)\right\rangle$ is the zero-temperature occupation of a quasiparticle with quantum 
number $\gamma$. We can now write

$$
\begin{aligned}
& \left\langle N\left|\sum_{M_{-}} \frac{\mathcal{V}^{+}\left|M_{-}\right\rangle\left\langle M_{-}\right| \mathcal{V}^{-}}{E_{M_{-}}-E_{0}}\right| N^{\prime}\right\rangle \\
& =\sum_{m_{-}} \sum_{\alpha \alpha^{\prime}, \mathbf{i i}^{\prime}}\left[1-n_{f}(\alpha)\right] \\
& \quad \times \frac{V_{\alpha, \mathbf{i}}^{*} V_{\alpha^{\prime}, \mathbf{i}^{\prime}}}{E_{m_{-}}-E_{0}+\epsilon_{\alpha^{\prime}}}\left\langle\psi_{f}(0)\left|f_{\alpha} f_{\alpha^{\prime}}^{\dagger}\right| \psi_{f^{\prime}}(0)\right\rangle \\
& \quad \times\left\langle n\left|d_{\mathbf{i}}^{\dagger}\right| m_{-}\right\rangle\left\langle m_{-}\left|d_{\mathbf{i}^{\prime}}\right| n^{\prime}\right\rangle .
\end{aligned}
$$

A similar expression can be obtained for the matrix elements involving states $\left|M_{+}\right\rangle$,

$$
\begin{aligned}
& \sum_{M_{+}}\left\langle N\left|\frac{\mathcal{V}^{-}\left|M_{+}\right\rangle\left\langle M_{+}\right| \mathcal{V}^{+}}{E_{M+}-E_{0}}\right| N^{\prime}\right\rangle=\sum_{m_{+}} \sum_{\alpha \alpha^{\prime}, \mathbf{i i}^{\prime}} n_{f}(\alpha) \\
& \quad \times \frac{V_{\alpha, \mathbf{i}} V_{\alpha^{\prime}, \mathbf{i}^{\prime}}^{*}}{E_{m_{+}}-E_{0}-\epsilon_{\alpha}}\left\langle\psi_{f}(0)\left|f_{\alpha}^{\dagger} f_{\alpha^{\prime}}\right| \psi_{f^{\prime}}(0)\right\rangle \\
& \quad \times\left\langle n\left|d_{\mathbf{i}}\right| m_{+}\right\rangle\left\langle m_{+}\left|d_{\mathbf{i}^{\prime}}^{\dagger}\right| n^{\prime}\right\rangle .
\end{aligned}
$$

Now, it is straightforward to show that the addition of Eqs. (A4) and (A5) leads to the final expression (5).

\section{APPENDIX B: TUNNELING TRANSITION RATES}

As stated in the Sec. II B, the cotunneling transition rates can be calculated applying the Fermi golden rule to the effective tunneling Hamiltonian $\mathcal{H}_{\text {cotun. Introducing the density of states }}$ $\rho_{\eta \sigma}$ and using Eqs. (A4) and (A5), the transition rate from a state $n$ of the central island to a state $n^{\prime}$, with the transport electron going from electrode $\eta$ to $\eta^{\prime}$ and its spin from $\sigma$ to $\sigma^{\prime}$, is given by

$$
\begin{aligned}
W_{n, n^{\prime}}^{\eta \sigma \eta^{\prime} \sigma^{\prime}}= & \frac{2 \pi}{\hbar} \int d \epsilon \rho_{\eta \sigma}(\epsilon) \rho_{\eta \sigma^{\prime}}\left(\epsilon+\Delta_{n n^{\prime}}\right) f\left(\epsilon-\mu_{\eta}\right) \\
& \times\left[1-f\left(\epsilon+\Delta_{n n^{\prime}}-\mu_{\eta^{\prime}}\right)\right] \\
& \times \mid\left\langle n\left|\hat{\mathcal{O}}_{\eta \sigma, \eta^{\prime} \sigma^{\prime}}^{(+)}(\epsilon, \epsilon+\Delta)\right| n^{\prime}\right\rangle \\
& -\left.\left\langle n^{\prime}\left|\hat{\mathcal{O}}_{\eta^{\prime} \sigma^{\prime}, \eta \sigma}^{(-)}(\epsilon+\Delta, \epsilon)\right| n\right\rangle\right|^{2}
\end{aligned}
$$

with $\Delta_{n n^{\prime}}=E_{n}-E_{n^{\prime}}$ and $f(\epsilon)$ the Fermi-Dirac distribution. The matrix elements of the $\hat{\mathcal{O}}^{( \pm)}$operators in Eq. (B1) are defined as

$$
\left\langle n\left|\hat{\mathcal{O}}_{\eta \sigma, \eta^{\prime} \sigma^{\prime}}^{(+)}\left(\epsilon, \epsilon^{\prime}\right)\right| n^{\prime}\right\rangle=\sum_{i i^{\prime}, m_{+}} \frac{V_{\eta, i}(\epsilon) V_{\eta^{\prime}, i^{\prime}}^{*}\left(\epsilon^{\prime}\right)}{E_{m_{+}}-E_{0}-\epsilon} \gamma_{n n^{\prime}}^{m_{+}}\left(i i^{\prime}, \sigma \sigma^{\prime}\right)
$$

and

$$
\left\langle n\left|\hat{\mathcal{O}}_{\eta \sigma, \eta^{\prime} \sigma^{\prime}}^{(-)}\left(\epsilon, \epsilon^{\prime}\right)\right| n^{\prime}\right\rangle=\sum_{i i^{\prime}, m_{-}} \frac{V_{\eta, i}^{*}(\epsilon) V_{\eta^{\prime}, i^{\prime}}\left(\epsilon^{\prime}\right)}{E_{m_{-}}-E_{0}+\epsilon^{\prime}} \gamma_{n n^{\prime}}^{m_{-}}\left(i i^{\prime}, \sigma \sigma^{\prime}\right),
$$

where we have used a simplified notation $V_{\eta, i}(\epsilon) \equiv V_{k(\epsilon) \eta, i}$. These transitions rates are in perfect agreement with the rates obtained by a second-order truncation of the $T$ matrix ${ }^{37,38}$
${ }^{1}$ B. C. Stipe, M. A. Rezaei, and W. Ho, Science 280, 1732 (1998).

${ }^{2}$ A. J. Heinrich, J. A. Gupta, C. P. Lutz, and D. M. Eigler, Science 306, 466 (2004).

${ }^{3}$ C. F. Hirjibehedin, C. P. Lutz, and A. J. Heinrich, Science 312, 1021 (2006).

${ }^{4}$ C. Hirjibehedin, C.-Y. Lin, A. Otte, M. Ternes, C. P. Lutz, B. A. Jones, and A. J. Heinrich, Science 317, 1199 (2007).

${ }^{5}$ A. F. Otte, M. Ternes, K. von Bergmann, S. Loth, H. Brune, C. P. Lutz, C. F. Hirjibehedin, and A. J. Heinrich, Nature Phys. 4, 847 (2008).

${ }^{6}$ S. Loth, K. von Bergmann, M. Ternes, A. F. Otte, C. P. Lutz, and A. J. Heinrich, Nature Phys. 6, 340 (2010).

${ }^{7}$ S. Loth, C. P. Lutz, and A. J. Heinrich, New J. Phys. 12, 125021 (2010).

${ }^{8}$ X. Chen, Y.-S. Fu, S.-H. Ji, T. Zhang, P. Cheng, X.-C. Ma, X.-L. Zou, W.-H. Duan, J.-F. Jia, and Q.-K. Xue, Phys. Rev. Lett. 101, 197208 (2008).

${ }^{9}$ N. Tsukahara, K. Noto, M. Ohara, S. Shiraki, N. Takagi, Y. Takata, J. Miyawaki, M. Taguchi, A. Chainani, S. Shin et al., Phys. Rev. Lett. 102, 167203 (2009).

${ }^{10}$ Y. S. Fu, T. Zhang, S. H. Ji, X. Chen, X. C. Ma, J. F. Jia, and Q. K. Xue, Phys. Rev. Lett. 103, 257202 (2009).

${ }^{11}$ Y.-S. Fu, S.-H. Ji, T. Zhang, X. Chen, X.-C. Ma, J.-F. Jia, and Q.-K. Xue, Appl. Phys. Lett. 95, 063107 (2009).

${ }^{12}$ A. A. Khajetoorians, B. Chilian, J. Wiebe, S. Schuwalow, F. Lechermann, and R. Wiesendanger, Nature (London) 467, 1084 (2010).

${ }^{13}$ S. Loth, M. Etzkorn, C. P. Lutz, D. M. Eigler, and A. J. Heinrich, Science 329, 1628 (2010).

${ }^{14}$ J. A. Appelbaum, Phys. Rev. 154, 633 (1967).
${ }^{15}$ J. Fernández-Rossier, Phys. Rev. Lett. 102, 256802 (2009).

${ }^{16}$ J. Fransson, Nano Lett. 9, 2414 (2009).

${ }^{17}$ M. Persson, Phys. Rev. Lett. 103, 050801 (2009).

${ }^{18}$ F. Delgado, J. J. Palacios, and J. Fernández-Rossier, Phys. Rev. Lett. 104, 026601 (2010).

${ }^{19}$ J. Fransson, O. Eriksson, and A. V. Balatsky, Phys. Rev. B 81, 115454 (2010).

${ }^{20}$ R. Žitko and J. Bonča, New J. Phys. 12, 063040 (2010).

${ }^{21}$ B. Sothmann and J. König, New J. Phys. 12, 083028 (2010).

${ }^{22}$ F. Delgado and J. Fernández-Rossier, Phys. Rev. B 82, 134414 (2010).

${ }^{23}$ D. V. Averin and Y. V. Nazarov, Phys. Rev. Lett. 65, 2446 (1990).

${ }^{24}$ D. V. Averin and Y. V. Nazarov, in Single Charge Tunneling: Coulomb Blockade Phenomena in Nanostructures, edited by H. Grabert and M. H. Devoret (Plenum Press and NATO Scientific Affairs Division, New York, 1992).

${ }^{25}$ P. W. Anderson, Phys. Rev. Lett. 17, 95 (1966).

${ }^{26}$ J. R. Schrieffer and P. A. Wolff, Phys. Rev. 149, 491 (1966).

${ }^{27}$ P. Recher, E. V. Sukhorukov, and D. Loss, Phys. Rev. Lett. 85, 1962 (2000).

${ }^{28} \mathrm{M}$. R. Wegewijs and Y. V. Nazarov, e-print arXiv:cond-mat/0103579.

${ }^{29}$ P. R. Bas and A. A. Aligia, J. Phys. Condens. Matter 22, 025602 (2010).

${ }^{30}$ A. Messiah, Quantum Mechanics (Dover, New York, 1999).

${ }^{31}$ C. Cohen-Tannoudji, G. Grynberg, and J. Dupont-Roc, AtomPhoton Interactions (Wiley and Sons, Inc., New York, 1998).

${ }^{32}$ O. Parcollet and X. Waintal, Phys. Rev. B 73, 144420 (2006).

${ }^{33}$ J. Merino and O. Gunnarsson, Phys. Rev. B 69, 115404 (2004). 
${ }^{34}$ C. W. J. Beenakker, Phys. Rev. B 44, 1646 (1991).

${ }^{35}$ F. Elste and C. Timm, Phys. Rev. B 71, 155403 (2005).

${ }^{36}$ F. Elste and C. Timm, Phys. Rev. B 75, 195341 (2007).

${ }^{37}$ T. Hansen, V. Mujica, and M. A. Ratner, Nano Lett. 8, 3525 (2008).

${ }^{38}$ N. Roch, R. Vincent, F. Elste, W. Harneit, W. Wernsdorfer, C. Timm, and F. Balestro, Phys. Rev. B 83, 081407 (2011).

${ }^{39}$ S. G. Tikhodeev and H. Ueba, in Problems of Condensed Matter Physics: Quantum Coherence Phenomena in Electron-hole and Coupled Matter-light Systems, edited by A. L. Ivanov and S. G. Tikhodeev (Oxford Science Publications, 2008), pp. 323-347.

${ }^{40}$ R. Žitko and J. Bonča, Phys. Rev. B 74, 045312 (2006).

${ }^{41}$ A. V. Balatsky, Y. Manassen, and R. Salem, Phys. Rev. B 66, 195416 (2002).

${ }^{42}$ J.-X. Zhu and A. V. Balatsky, Phys. Rev. B 67, 174505 (2003).

${ }^{43}$ Z. Nussinov, M. F. Crommie, and A. V. Balatsky, Phys. Rev. B 68, 085402 (2003).

${ }^{44}$ S. Datta, Quantum Transport: Atom to Transistor (Cambridge University Press, Cambridge, 2005).

${ }^{45}$ J.-P. Gauyacq, F. D. Novaes, and N. Lorente, Phys. Rev. B 81, 165423 (2010).

${ }^{46}$ F. D. Novaes, N. Lorente, and J.-P. Gauyacq, Phys. Rev. B 82, 155401 (2010).
${ }^{47}$ N. Lorente and J.-P. Gauyacq, Phys. Rev. Lett. 103, 176601 (2009).

${ }^{48}$ A. N. Rudenko, V. V. Mazurenko, V. I. Anisimov, and A. I. Lichtenstein, Phys. Rev. B 79, 144418 (2009).

${ }^{49}$ G. Racah, Phys. Rev. 62, 438 (1942).

${ }^{50}$ M. J. Frisch, G. W. Trucks, H. B. Schlegel, G. E. Scuseria, M. A. Robb, J. R. Cheeseman, G. Scalmani, V. Barone, B. Mennucci, G. A. Petersson et al., Gaussian09, Revision A.1 (Gaussian, Inc., Wallingford, CT, 2009).

${ }^{51}$ I. Schnell, G. Czycholl, and R. C. Albers, Phys. Rev. B 68, 245102 (2003).

${ }^{52}$ D. Jacob, K. Haule, and G. Kotliar, Phys. Rev. Lett. 103, 016803 (2009).

${ }^{53}$ E. Dagotto, Nanoscale Phase Separation and Colossal Magnetoresistance (Springer-Verlag, Berlin, 2003).

${ }^{54}$ M. G. Zhao and M. Chiu, Phys. Rev. B 52, 10043 (1995).

${ }^{55}$ L. Zhou, J. Wiebe, S. Lounis, E. Vedmedenko, F. Meier, S. Blügel, P. H. Dederichs, and R. Wiesendanger, Nature Phys. 6, 187 (2010).

${ }^{56}$ J. C. Slater and G. F. Koster, Phys. Rev. 94, 1498 (1954).

${ }^{57}$ N. W. Ashcroft and N. D. Mermin, Solid State Physics (Thomson Learning, 1976).

${ }^{58}$ A. Kogan, S. Amasha, D. Goldhaber-Gordon, G. Granger, M. A. Kastner, and H. Shtrikman, Phys. Rev. Lett. 93, 166602 (2004). 